Vol.02/ No. 02

Pages: 91-99

https://www.irojournals.com/itdw/

DOI: https://doi.org/10.36548/jitdw.2020.2.002

\title{
All India Grievance Redressal App
}

\author{
${ }^{1}$ Viral Patel, ${ }^{2}$ Daanyaal Kapadia, ${ }^{3}$ Deval Ghevariya, ${ }^{4}$ Shiburaj Pappu \\ ${ }^{1}$ Student, Department of Computer Engineering, RCOE, MU, Mumbai \\ Email: viral786patel@gmail.com \\ ${ }^{2}$ Student, Department of Computer Engineering, RCOE, MU, Mumbai \\ Email: daanyaalkapadia13@gmail.com \\ ${ }^{3}$ Student, Department of Computer Engineering, RCOE, MU, Mumbai \\ Email: devalghevariya4183@gmail.com \\ ${ }^{4}$ Head of Department, Department of Computer Engineering, RCOE, MU, Mumbai \\ Email: shiburaj@eng.rizvi.edu.in
}

\begin{abstract}
Citizens of the India face civic problems in their day-to-day lives. They resort to the one of many ways provided by the government to file their complaints. The grievance registration systems have evolved in many ways with the advancement in technologies to simplify the task. This paper presents the architecture of a Grievance redresser Application where the civilians can address any kind of complain which they are facing. The main focus of the project is about the pothole related complains. One of the most difficult task for the government officials to estimate the total time and material required to fill the pothole of widely spaced roads, this is one of the major problem faced by government authorities which leads to delaying in repairing the pothole and increased the cost to fill a particular pothole. This Application will give easy access to people to put their complaints towards the government.
\end{abstract}

Keywords: Potholes, Complaints, Android, GPS location, Geocoding

\section{Introduction}

Efficient governance is one of the biggest challenges for the government of India. One of the important aspect of this governance is a robust complaint registration system through which citizens can express their grievances. In attempts to make the system accurate, efficient and easy to use, several new ways have been devised. The current system in India provides some of these ways for citizens to register their complaints: Online complaint registration portal, E-mail, Helpline number and Short Messaging Service (SMS). All the above methods require the complainant to describe the location of the complaint manually. This method is prone to human errors and thus misinterpretations can occur, thus this leading to inefficiency in addressing the complaints and the complaints gets null and void. The proposed All India Grievance Redressal App is developed with the aim of solving the day-to-day life problems of all the citizens of India through a mobile application. In the past decade, smart mobile devices have grown cheaper and have made their place in every common people pockets. The smartphones have many features like getting the GPS location, taking pictures, etc. which made us possible to come up with this idea.

All India Grievance Redressal App is developed using Java and XML (eXtensible Mark-up Language). In this app, Complaints are categorized into two type viz. Complaint Type and Complaint sub-type in 
Journal of Information Technology and Digital World (2020)

Vol.02/ No. 02

Pages: 91-99

https://www.irojournals.com/itdw/

DOI: https://doi.org/10.36548/jitdw.2020.2.002

which complaints are classified into multiple complaint types like Solid Waste Management, Drainage, Storm Water drain, Road and Traffic, Factories, Licence, Water Supplies and many more. Complaint sub-type is the further categorisation of the particular complaint type. Further the complaints will get divided with respect to location. That is state wise, if the complaint location is Maharashtra than the complaint will get directed to the Maharashtra state officials. State admins can view the complaints registered in their state and the number of users registered in that state in Website. Admin website is developed by express framework of Node JS (JavaScript) which uses passport JS for authentication and Bcrypt JS module is used for encryption and decryption of Password. Basic details of admins are stored in MongoDB (Database) Atlas database. There is one main admin who will manage and monitor all the state admins of the country. If Complaint solving rate is very less in particular state then main admin can communicate with state admin to discuss the issue. In order to place any complaint, user must be registered with the app by providing simple details like name, email, mobile number, username and password, etc. User data and complaint related data is stored in Firebase Real-time database. Firebase real-time database is NoSQL (No Structured Query Language) database where data is stored in keyvalue pairs. NoSQL database implementation is easy and one of the major advantage of NoSQL database is that is uses cheap servers to manage data and transitions while on the other hand Relational DBMS databases are expensive and it uses storage systems and big servers. So the storing and processing data in the case of NoSQL can be many times cheaper than the cost of RDBMS (Relational Database Management System) (cost per gigabyte). Our App uses Email and Password based authentication mechanism. The user will select the category then the sub-category and then the user will be allowed to enter a description about the complaint and the user can also upload the image of the complaint and image will be stored in Firebase Storage which is a powerful, simple, and cost-effective object storage service. Glide, the most popular image loading library is used to download the image files in the application. Picasso is also used for the same but Glide is more memory efficient (about 8 MB) than Picasso (about 13 MB). The Picasso loads the full-size image into the memory and relies on GPU (Graphics Processing Unit) to resize that image to fit into the size of the ImageView. We will require less memory if we use While Glide, as it only loads an image that is priory resized with respect to ImageView, unlike Picasso than loading the full image and require more memory. This helps in preventing errors like OutOfMemoryError. The complaint location will be automatically captured. After successful submission of complaint, complaint ID will be auto-generated and this is the unique complaint id with which user can access the complaint details. The feature of automatic location capturing will avoid the Human type errors and also save the time of user.

\section{Related Works}

\subsection{Survey Existing system}

Vishesh K. Kandhari et.al [1] have developed an architecture for a grievance mobile application in which they have proposed that when the user registers any complain the current location of the user is fetched using the Google Maps API. So the misinterpretation from the user side could be minimized. No extra hardware required except the mobile phone itself. The second thing that they proposed is that they will use a priority based system if suppose complain is not addressed in a particular span of time then the complaint is forwarded to the senior authority. 
Journal of Information Technology and Digital World (2020)

Vol.02/ No. 02

Pages: 91-99

https://www.irojournals.com/itdw/

DOI: https://doi.org/10.36548/jitdw.2020.2.002

Dhananjay R. Kalband, Priyanka Kulkarni, Uma Nagarseka and Aditi Mhapsekar et.al [2] have developed a system for Speech enabled Android application for road and traffic related complaint using GSM-SMS and GPS technology. This application uses a method of speech to text to describe the complaint. The GPS coordinates is generated by the system and then it is added at the end of the complaint information and sends the data over the GSM network in plain text message format (SMS) to the server. The SMS server then retrieves all the information and stores it in the database. The information is then plotted on a map with the help of web interface. Umar Farooq et.al [3] has also done similar kind of work in this paper.

Yoon Yeh Tan, Yin Ping Ng, Kim Nee Goh, Yoke Yie Chen and Kamaruzaman Jusoff et.al [4] have developed a system for road management. In this proposed system obtains GPS coordinates on a smartphone and then the complaint along with the description and the GPS information is send via an SMS to the server. The data is then retrieved and saved in the database. This obtained information is then plotted on Google Maps.

Amol Madhav Khatkhate et.al [5] have developed an algorithm for getting the time required to fill a particular pothole as well as the amount of material required. The only inputs required is the extreme $x$ and y co-ordinates of the pothole (contour). Aaditeshwar Seth et.al [6] has discussed application of mobile and social media in public grievance.

\subsection{Limitation of the Existing System}

In the different system we saw above in the survey existing system, the first paper [1] does not consist of the pothole mechanism that is it can't calculate the time and the amount of material required to fill a particular pothole. This is not giving to speed up the governance process especially in the finance sector regarding the potholes. The second and the third system is the SMS related system which does not consist of any mobile application. Which in then can lead to miss interpretation of information in the complaint and that can lead the complaint to null and void although the complaints are plotted on Google Maps. [2][4]. the [5] paper have developed an algorithm but not implemented in the real software. That is only the mechanical point of view to the problem.

\subsection{Problem Statement and Objective}

The biggest problem of India is population which leads to major problems like water logging, water leakage, street light problem, channel problem, pothole on road problem, garbage etc. One of the major problem is that government doesn't know how much cost is required before seeing the pothole, our app will enable to estimate the cost required of a particular pothole. These are some of the major problems which our app will cover.

\section{Proposed Work}

With the help of android application, any user can file any type of Complaint across all the states of the India. Again, Complaints are classified into Complaint type and Complaint subtype. For example, Roads and Traffic is main Complaint type and under Roads and Traffic different sub-complaints have been included like Potholes, Street Light Problem, etc. The main peculiarity of the app is that while registering any complaint, complaint location is automatically captured in the form of Latitude and Longitude co-ordinates. Geocoding Process is used to convert the location's latitude and longitude values into further details about an address and vice versa. Both forward and backward geocoding is 
Journal of Information Technology and Digital World (2020)

Vol.02/ No. 02

Pages: 91-99

https://www.irojournals.com/itdw/

DOI: https://doi.org/10.36548/jitdw.2020.2.002

possible. Users will send Complaints from Mobile Application. Complaint location will be automatically captured which will avoid the Human type errors and save the Time of User. As shown in figure 1 and figure 2.



Figure 1



Figure 2

Workers of different states can login through the app and the location will be automatically captured as shown in figure 3 and with respect to the zip code of the location the complaint will be displayed to the worker and worker can check the Complaints received in particular Region of State. Workers will solve the complaint and Send the details of Solved Complaints including Image of Solved Complaint to the Admin as shown in figure 4.



\section{COMPLAINT DESCRIPTION}
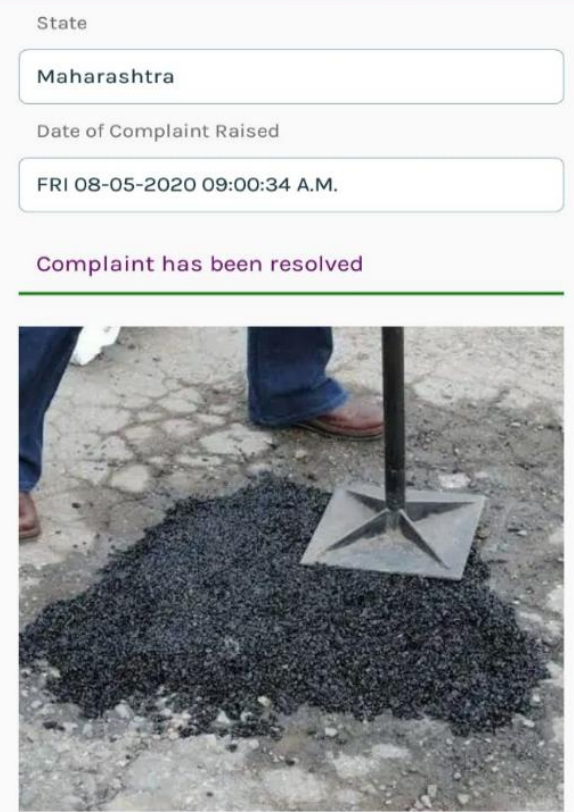
Journal of Information Technology and Digital World (2020)

Vol.02/ No. 02

Pages: 91-99

https://www.irojournals.com/itdw/

DOI: https://doi.org/10.36548/jitdw.2020.2.002

Figure 3

Figure 4.

Users can search the complaints by using Complaint ID or Complaint Type in their Mobile through App as shown in figure 7 and further user can navigate through solved and unsolved complaints which can be viewed in both ascending as well as in descending order as shown in the figure as shown in figure 5 $\& 6$.

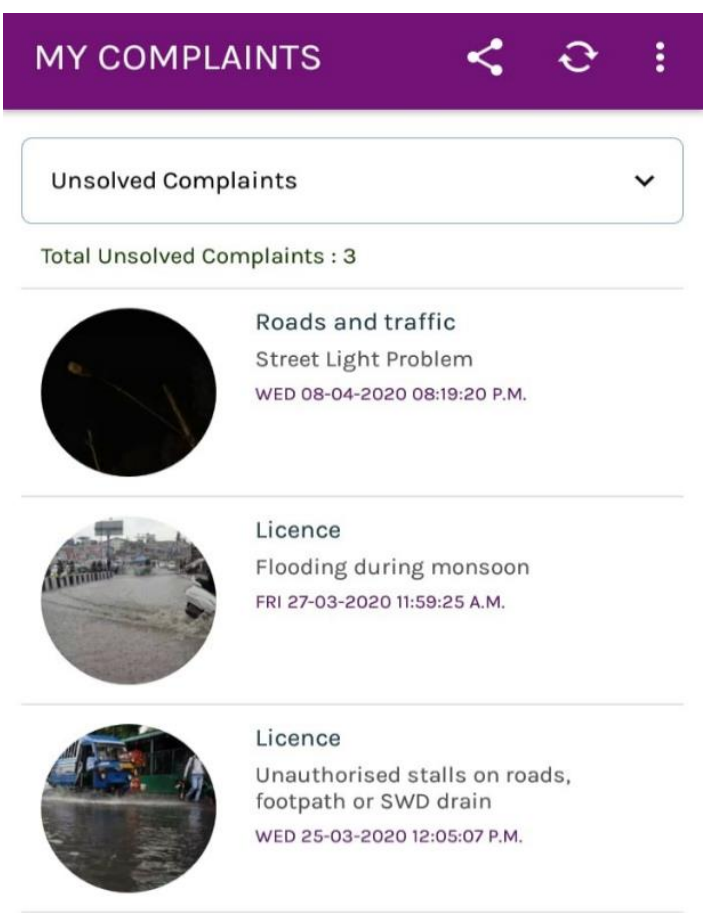

Figure 5

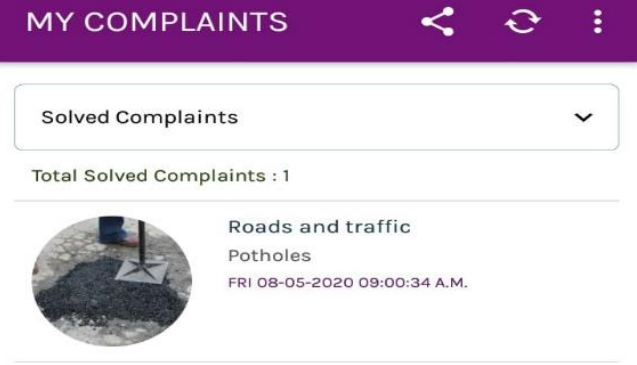

Figure 6

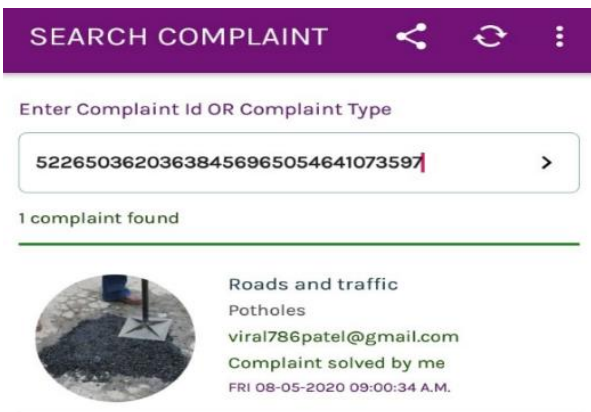

Figure 7

In the web based Admin panel there are different logins for different state and one main login for the entire county. After logging into the admin panel, here the complaints are divided into three categories namely pending complaints, partially solved complaints and solved complaints. In pending complaints only those complaints will be displayed which are filed by the user and no further action is taken as shown in figure 8. 
Journal of Information Technology and Digital World (2020)

Vol.02/ No. 02

Pages: 91-99

https://www.irojournals.com/itdw/

DOI: https://doi.org/10.36548/jitdw.2020.2.002

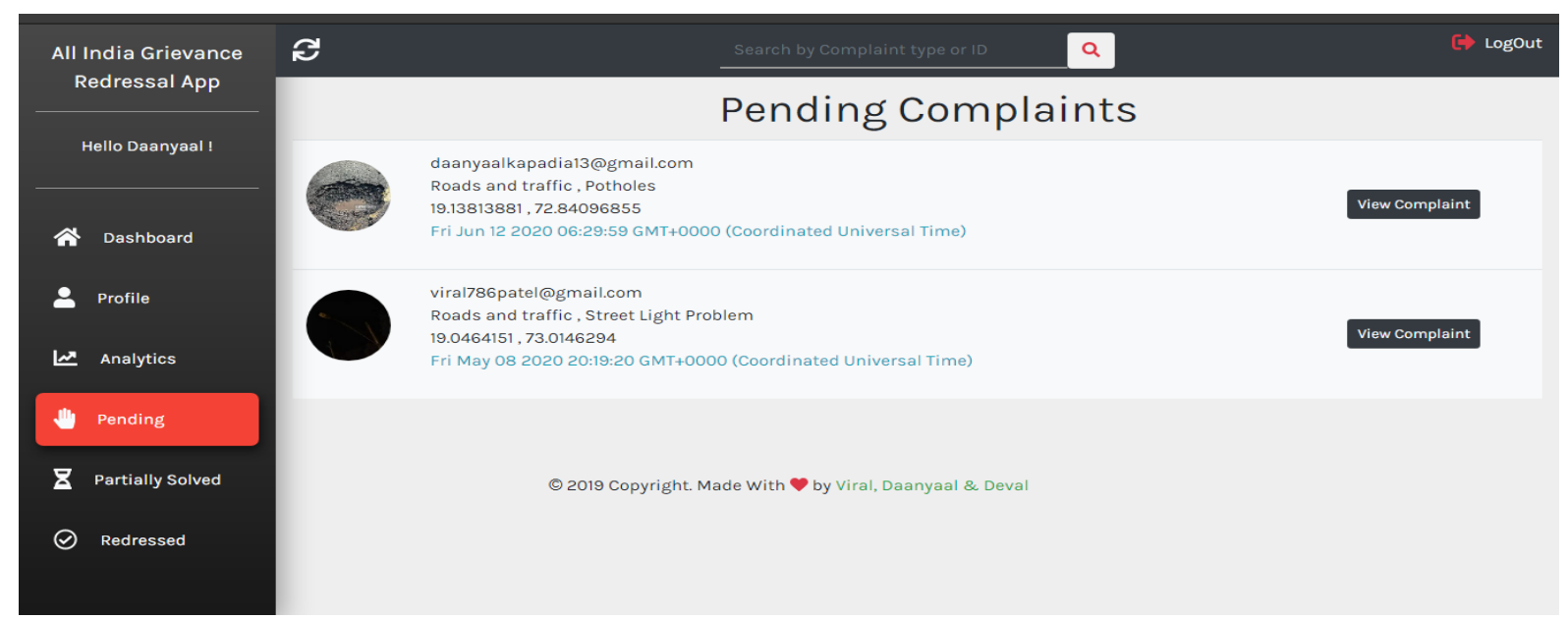

Figure 8

The partially solved complaints are those complaint which are solved by the worker and from the admin side the complaint needs to be checked weather the complaints is solved properly or if any changes are needed to be made. The admin will check through the image provided from the worker and if ever this is correct the admin will solve the complaint by clicking on the solve button as shown in the figure 9 and a notification to the worker and user will be generated and a mail will be sent to the user as will along all the complaint details.

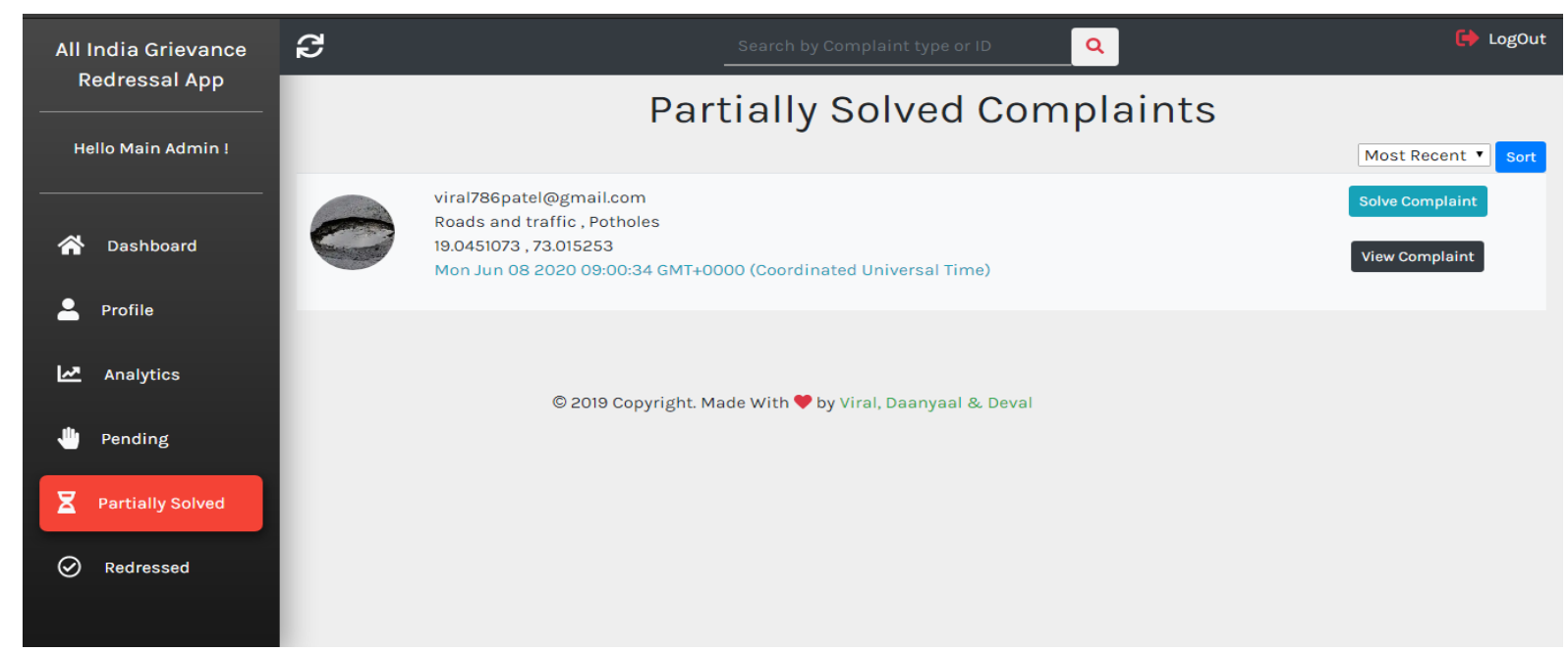

Figure 9

The solved complaints are those complaints which are solved by the admin from the web application after cross checking the solved image which is provided by the worker when the worker solves the complaint (see figure 10). The image which is displayed here is the solved image of the complaint. This solved complaint will now be displayed in the solved section in the user application. Partially solved and pending both will be displayed as pending in the user app. 
Journal of Information Technology and Digital World (2020)

Vol.02/ No. 02

Pages: 91-99

https://www.irojournals.com/itdw/

DOI: https://doi.org/10.36548/jitdw.2020.2.002

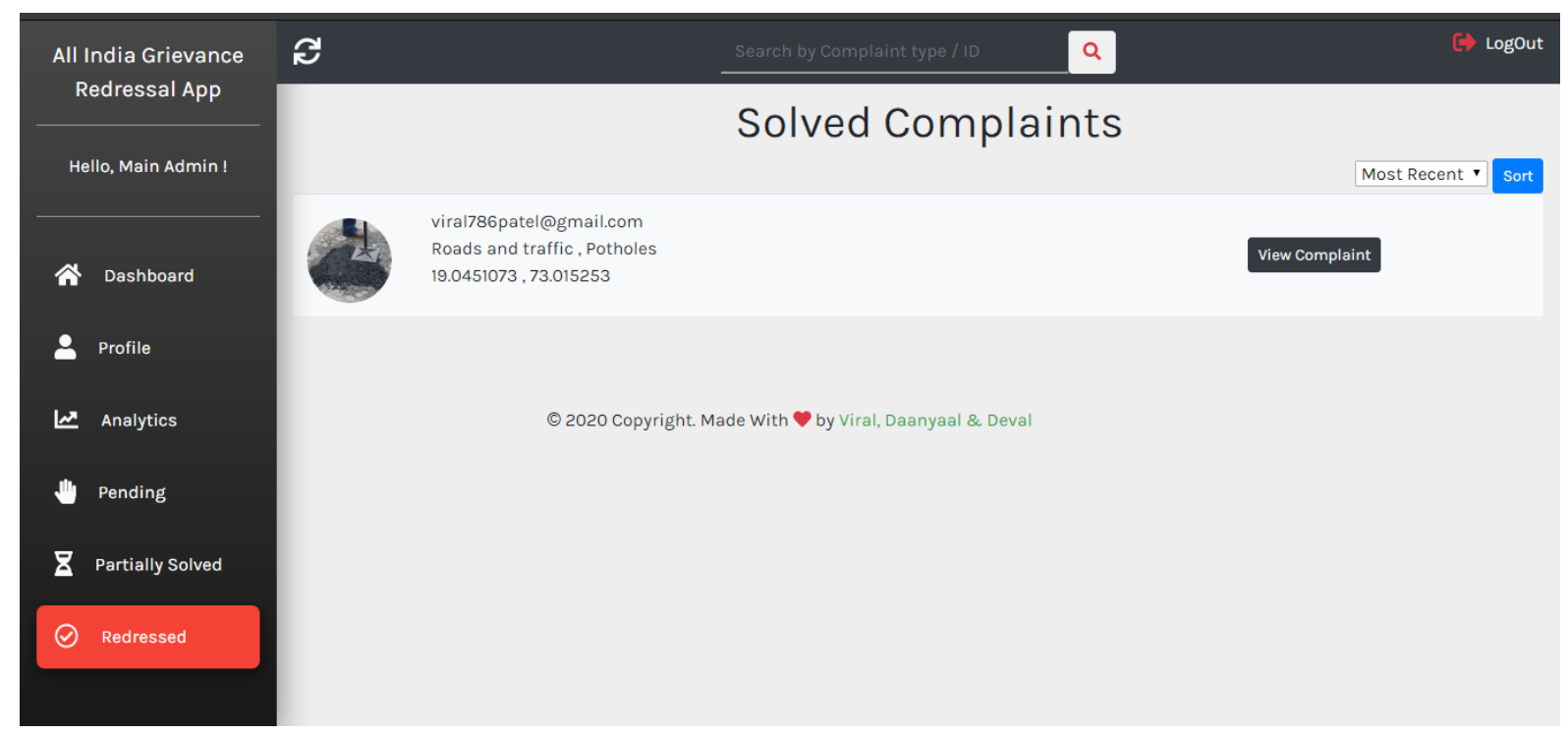

Figure 10

\section{Extraction the Time and Amount of Material Required to Fill a Pothole}

We have prepared an algorithm in which we can estimate the time required to fill a particular pothole and also estimates the amount of material required to fill the pothole. The algorithm in implemented in MatLab. The admin can take the images from website and can run the algorithm on it to calculate the time and amount required.

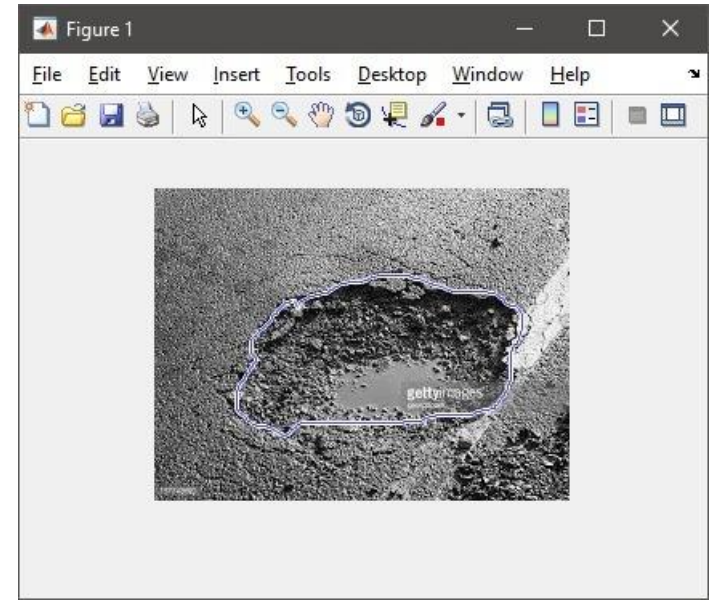

Figure 11

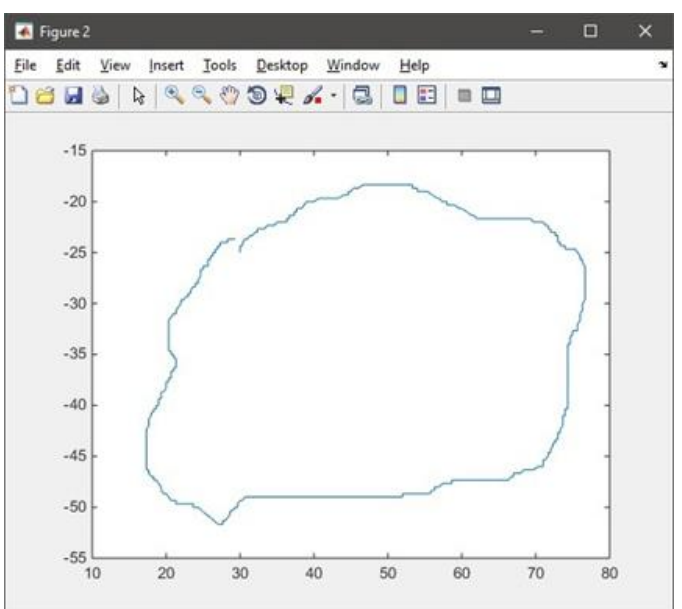

Figure 12

After receiving any pothole related complaint, admin will mark the contour around the pothole as shown in the Fig.11. Marking of the contour is done using myfreehand function in Matlab. After marking of the contour, the contour will be displayed in a blank sheet on a new window as shown in Fig. 12. After this the max and minimum coordinates of the contour will be marked and considering the max and min coordinates the calculations will be done and the result will be display. This data will be given to workers who are operating in the region from where the complaint has been raised. 
Journal of Information Technology and Digital World (2020)

Vol.02/ No. 02

Pages: 91-99

https://www.irojournals.com/itdw/

DOI: https://doi.org/10.36548/jitdw.2020.2.002

\subsection{Algorithm}

- First find the maximum and minimum coordinate of given potholes in Y direction.

- Divide given pothole into no of Y steps by dividing the distance between the maximum and minimum Y coordinates with the size of nozzle.

- Then create array of X co-ordinates for each Y step.

- Use $\min (\mathrm{X})$ to find the closest point from each array and denote it's by Index.

- Eliminate this point from the original array.

- Recompute the second closest point using $\min (\mathrm{X})$ function and denote it by IndexNew.

- No of X steps $=$ abs $((X I n d e x-X I n d e x n e w) /$ Nozzle size $)$.

- Find the total time required to fill potholes based on above calculation.

- Material required that also can be calculated.

\section{Conclusion and Future Work}

Hence in the paper we have proposed and implement an idea to file the grievance of the people in a very autonomous way. The user only needs a smart phone to file the complaint and nothing else, as most to the people have the access to the smartphones and internet in the country so the proposed project can be a success. As in the project the android application is used for filing the complaint and the website acts as an admin panel. We have also implemented a MATLAB algorithm for pothole related complaint with which we can calculate the amount of material required and the time required to fill a particular complaint.

We can further improve this project by filing any complaint related to road and traffic through CCTV cameras which are installed on the road or installing cameras on the government vehicles. These cameras will automatically detect the pothole or any other complaints with the help of machine learning algorithm. On finding the pothole or other grievance the complaint will be automatically stored in the database with the latitude and longitude of the complaint and image of it. We can also add a chat bot in the android application for user's assistance. We can also develop a robot for pothole related complaints which will act as a worker which will automate the task, the robot will get the amount of material and time required to fill the pothole, so by placing the robot on the complaint location the robot will itself fill the pothole. And there can be many other thing which can be added to this project to enhance the scalability, reliability, efficiency of the system.

\section{References}

[1] Vishesh K. Kandhari and Keertika D. Mohinani. "GPS based Complaint Redressal System" 2014 IEEE Global Humanitarian Technology Conference - South Asia Satellite (GHTC-SAS) September 26-27, 2014 Trivandrum 
Journal of Information Technology and Digital World (2020)

Vol.02/ No. 02

Pages: 91-99

https://www.irojournals.com/itdw/

DOI: https://doi.org/10.36548/jitdw.2020.2.002

[2] Aditi Mhapsekar, Uma Nagarseka, Priyanka Kulkarni and Dhananjay R. Kalbande. "Voice enabled Android application for vehicular complaint system using GPS and GSM-SMS technology," in World Congress on Information and Communication Technologies, 2012, pp. 520-524.

[3] Umar Farooq, Tanveer ul Haq, Muhammad Amar, Muhammad Usman Asad and Asim Iqbal. "GPSGSM Integration for Enhancing Public Transportation Management Services" in Second International Conference on Computer Engineering and Applications, 2010, pp. 142147.

[4] Kim Nee Goh, Yin Ping Ng, Kamaruzaman Jusoff, Yoke Yie Chen and Yoon Yeh Tan. "Architecture of a GPS-Based Road Management System," World Applied Sciences Journal 12 (Special Issue on Computer Applications and Knowledge Management), pp. 26-31, 2011.

[5] Qureshi, Aftab, Yadav Vivek, Shaikh, Nadeem, Shivani, Tabish, Khatkhate, Amol PY "Simulation of Pothole Filling Machine" International Conference on Global Technology Initiatives, At Mumbai

[6] Aaditeshwar Seth, Abhishek Katyal, Rohit Bhatia. "Application of Mobile Phones and Social Media to Improve Grievance Redressal in Public Services” Department of Computer Science IIT Delhi. 